\title{
Traqueobroncopatía osteocondroplástica: reporte de dos casos y revisión de la literatura
}

PATRICIO RIOSECO S.*, DANIELA YÉVENES A.** y JOSÉ SCHALPER P.***

Tracheobronchopathia osteochondroplastica: presentation of two cases

Tracheobronchopathia osteochondroplastica is a rare disease characterized by oteocartilagenous tissue bulking from the submucous layer into the lumen of large airways. We present two patients whose etiologic study for unrelated reasons rendered characteristic findings of this pathology. The literature about this disease is reviewed.

Key words: Tracheobronchopathia, osteochondroplastica, tracheal nodules, hemoptysis.

\section{Resumen}

La traqueobroncopatía osteocondroplástica es una enfermedad poco frecuente, caracterizada por la proliferación de tejido óseo y cartilaginoso en la submucosa de las vías aéreas mayores. Presentamos dos casos de pacientes en cuyo estudio por distintos motivos etiológicos se encontraron lesiones compatibles con esta patología, y se revisa el tema.

Palabras clave: Traqueobroncopatía osteocondroplástica, nódulos traqueales, hemoptisis.

\section{Introducción}

La traqueobroncopatía osteocondroplástica (TOCP) es una enfermedad benigna, poco frecuente, caracterizada por la formación de nódulos de color blanco o blanco amarillentos de tejido óseo y cartilaginoso en la submucosa de la tráquea y ocasionalmente de los bronquios principales, respetando siempre la pared membranosa posterior. La incidencia real no se conoce ya que a menudo es asintomática o los síntomas son inespecíficos. Constituye habitualmente un hallazgo en los estudios de imágenes o endoscópicos que se realizan por otras patologías o en autopsias.

Presentamos dos casos de pacientes portadoras de TOCP que ilustran las características broncoscópicas, imagenológicas e histológicas del cuadro. Una de ellas presenta alteraciones funcionales y clínica, con diagnóstico realizado 19 años antes durante el estudio de una hemoptisis; y la segunda, como hallazgo incidental en el estudio de un mesotelioma pleural.

\section{Caso 1}

Mujer de 69 años, con antecedentes de diabetes mellitus tipo 2, reflujo gastroesofágico, colelitiasis, glaucoma, úlcera gástrica tratada, tuberculosis pulmonar dudosa a los 22 años y limitación crónica del flujo aéreo secundaria (LCFA). Ingresó por urgencias por neumonía izquierda de evolución tórpida; se hospitalizó con diagnóstico de insuficiencia respiratoria global y LCFA sobreinfectada, requiriendo manejo con ventilación no invasiva. Entre sus antecedentes, destacaba que en 1992, a causa de un episodio de hemoptisis fue estudiada con fibrobroncoscopía que describía lesiones nodulares de paredes anteriores y laterales que comprometían hasta bronquios lobares, con biopsia confirmatoria de TOCP. La evolución posterior fue tórpida, con infecciones recurrentes de vías aéreas. La evaluación otorrinolaringológica demostró rinitis atrófica y ocena. Funcionalmente presentaba una limitación obstructiva del flujo aéreo, sin variación significativa de los volúmenes durante sus años de evolución.

\footnotetext{
* Médico Broncopulmonar, Hospital Las Higueras. Talcahuano, Chile.

** Residente de Medicina Interna, Universidad de Concepción.

****Médico Patólogo, Jefe Unidad de Patología y Citopatología, Hospital Las Higueras.
} 
Durante su hospitalización la curva flujo/volumen (Figura 1) fue sugerente de obstrucción fija de vía aérea central. La tomografía de senos paranasales mostró imagen compatible con rinitis crónica atrófica (Figura 2). La tomografía computada evidenció calcificaciones desde la submucosa de tráquea, bronquios fuente y lobares, con deformación importante, respetando la pared posterior (Figura 3). La videobroncosco- pía mostró tráquea estrecha, infiltrada por tejido osteocartilaginoso que disminuye su lumen, carina con múltiples zonas de osificación y árbol bronquial con osificación de sus paredes anterior y laterales hasta los ramos subsegmentarios (Figura 4). La biopsia informó presencia de tejido cartilaginoso y óseo en la submucosa, con indemnidad de la mucosa, consistente con TOCP (Figura 5).

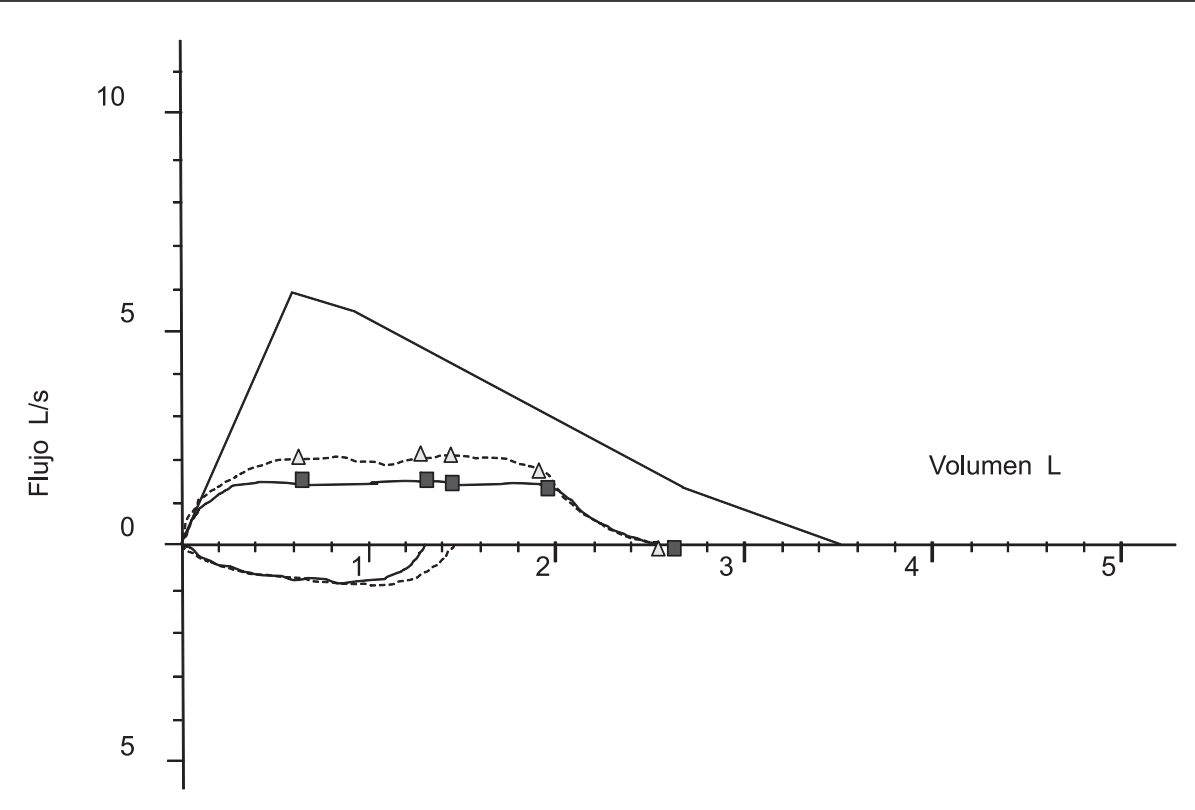

Figura 1. Curva flujo/volumen sugiere obstrucción fija de vía aérea central. Hay una marcada limitación inspiratoria y espiratoria tanto en condiciones basales (-ם-) como post-broncodilatador (- $\Delta-)$, con formación de una meseta.

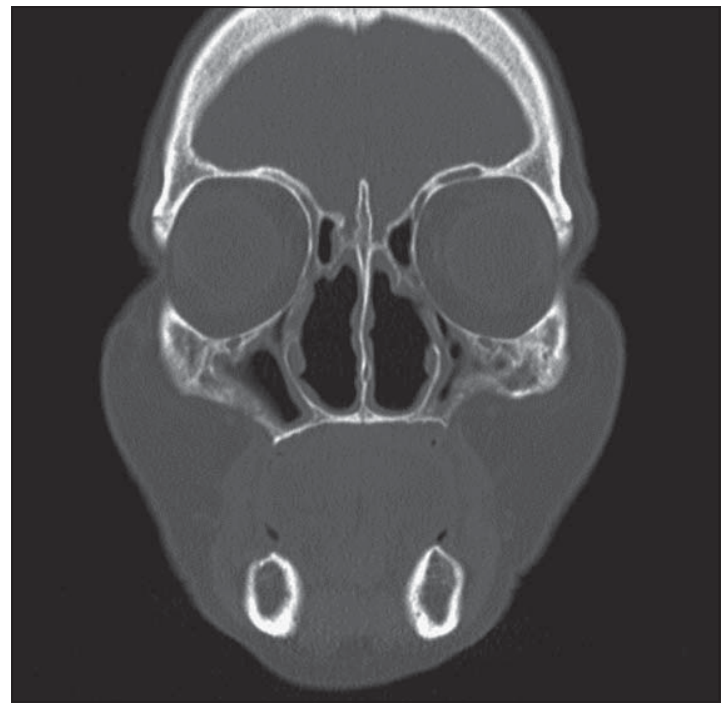

Figura 2. Tomografía axial computarizada de senos paranasales muestra rinitis crónica atrófica.

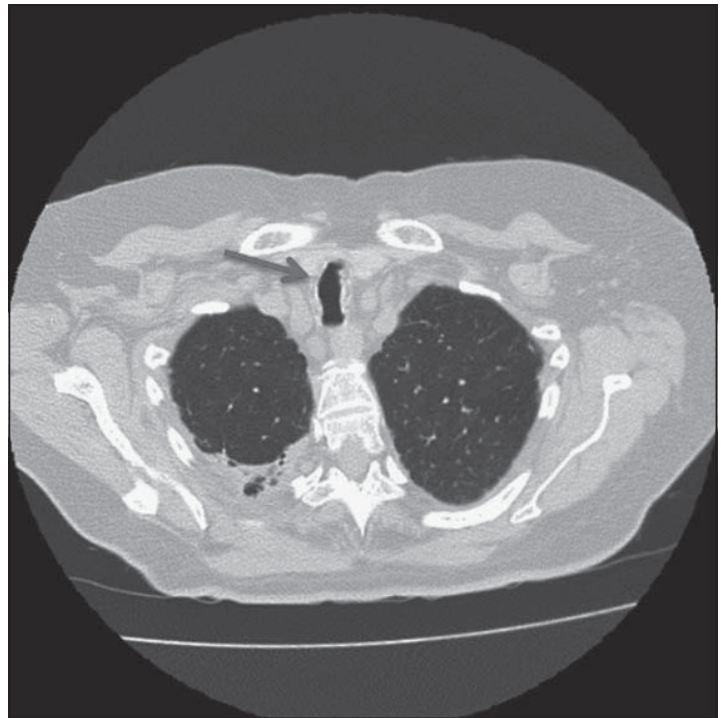

Figura 3. La tomografía de tórax muestra calcificaciones desde la submucosa de tráquea, bronquios fuente y lobares, con deformación importante, respetando la pared posterior (flecha). 


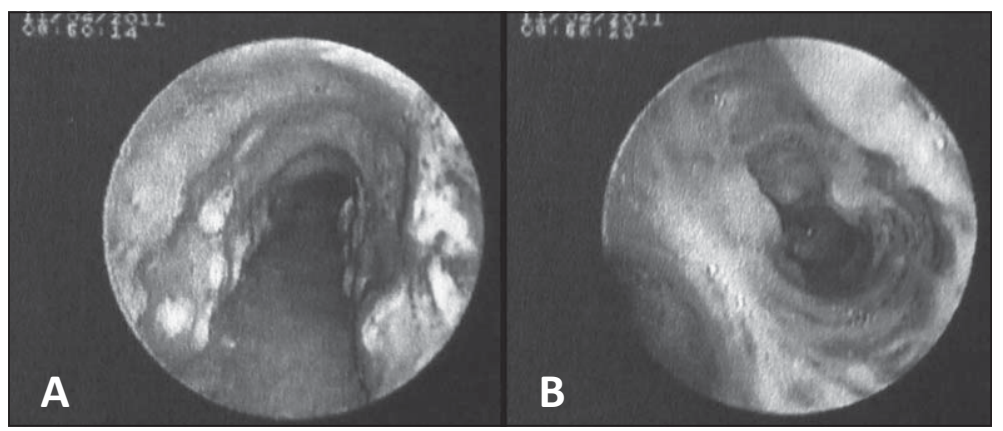

Figura 4. Videobroncoscopia. (A) Se observan nódulos osteocartilaginosos a nivel traqueal respetando pared posterior. (B) Se observa presencia de lesiones hasta ramos subsegmentarios.

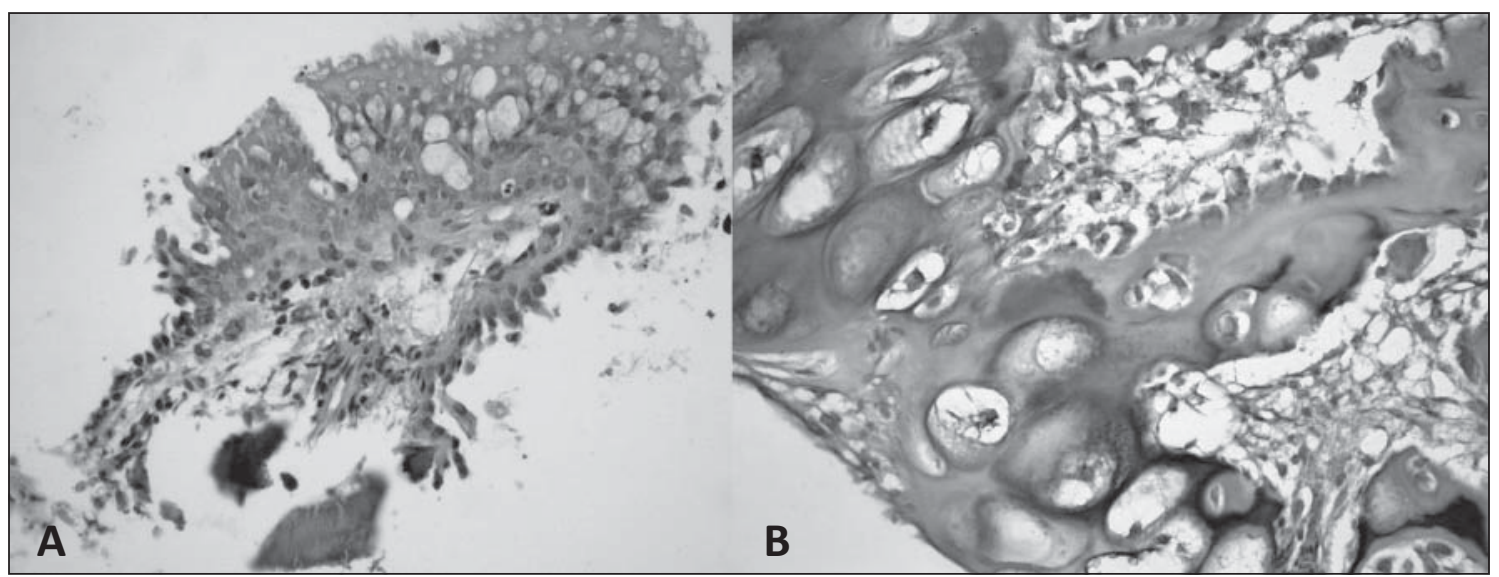

Figura 5. (A) Imagen de bronquio a 100X muestra indemnidad del epitelio, con presencia de tejido cartilaginoso y óseo en la submucosa. (B) En corte a 400X se observa presencia de médula ósea y tejido cartilaginoso en maduración.

\section{Caso 2}

Mujer de 69 años, con antecedentes de hipotiroidismo, colecistectomía, apendicectomía e infecciones urinarias repetidas asociadas a litiasis renal. Consultó en servicio de urgencias por cuadro de dolor dorsal y de hombro izquierdo, asociado a baja de peso (alrededor de 6 kilogramos en los últimos 4 meses). Se pesquisó cuadro clínico y radiológico compatible con neumonía basal izquierda, por lo que fue hospitalizada. Completado el tratamiento antibiótico, fue dada de alta, con control en policlínico de reumatología por mantener molestias en hombro izquierdo.

En su control reumatológico, nueva radiografía de tórax evidenció derrame encapsulado en base pulmonar izquierda, de características sospechosas de neoplasia, por lo que se hospitalizó. La tomografía de tórax (Figura 6) demostró engrosamiento protruyente calcificado de la pared lateral de la tráquea y engrosamiento pleural izquierdo, de aspecto neoplásico sugerente de mesotelioma. La biopsia de Cope fue negativa para células neoplásicas, y la videobroncoscopía (Figura 7) evidenció tráquea recta sembrada por mamelones blanquecinos duros, desde tercio superior hasta la carina. La histología demostró osificación de la mucosa bronquial consistente con TOCP. La paciente falleció antes de completar el estudio etiológico de su patología pleural.

\section{Discusión}

La TOCP constituye una entidad benigna que afecta las vías aéreas mayores, cuya progresión en el tiempo es muy lenta, pero que puede evolucionar desde un estado asintomático, hasta una enfermedad devastadora, causante de invalidez respiratoria secundaria a la obstrucción mecánica severa de la vía aérea central ${ }^{1} \mathrm{o}$ infectarse y causar falla respiratoria grave como en el primer caso.

Hasta la fecha se han comunicado alrededor de 400 casos, es probable que la cantidad de pacientes afectados sea mucho mayor ya que es una condición que se diagnostica de manera incidental en razón de intubación endotraqueal, 


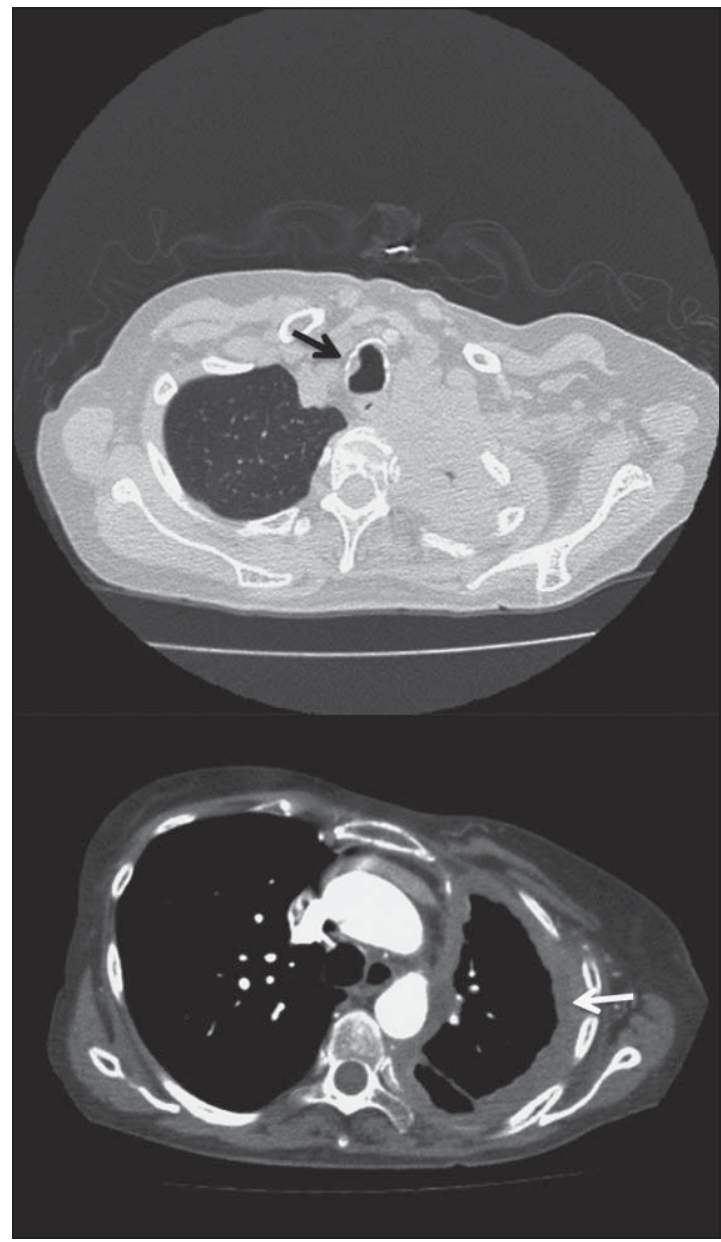

Figura 6. Imágenes de tomografía de tórax evidencian engrosamiento protruyente calcificado de la pared lateral de la tráquea (flecha negra) y engrosamiento pleural izquierdo (flecha blanca)

exámenes de imágenes o endoscópicos realizados por patologías no relacionadas, o en relación a complicaciones, como infecciones repetidas o hemoptisis ${ }^{2,3}$. Su prevalencia e incidencia real es infrecuente y los datos varían entre diferentes autores; así por ejemplo, en estudios de autopsia la incidencia fluctúa entre 1/400 a 3/1.000 mientras que en broncoscopías ésta varía entre $1 / 125$ a 1/6.000. Un autor ha comunicado una incidencia de 2 a 7 casos por 1.000 pacientes haciendo un cálculo sencillo a partir de hallazgos de autopsia en un centro y de hallazgos endoscópicos en otros dos centros reuniendo un total de 14 casos en 3.130 individuos ${ }^{4}$. Nosotros en 3.420 broncoscopías, hemos encontrado 2 casos con las características clásicas de la TOCP confirmados con histología. La patogenia tampoco está bien dilucidada, pero se reportan casos familiares, por lo que es posible que exista cierta predisposición

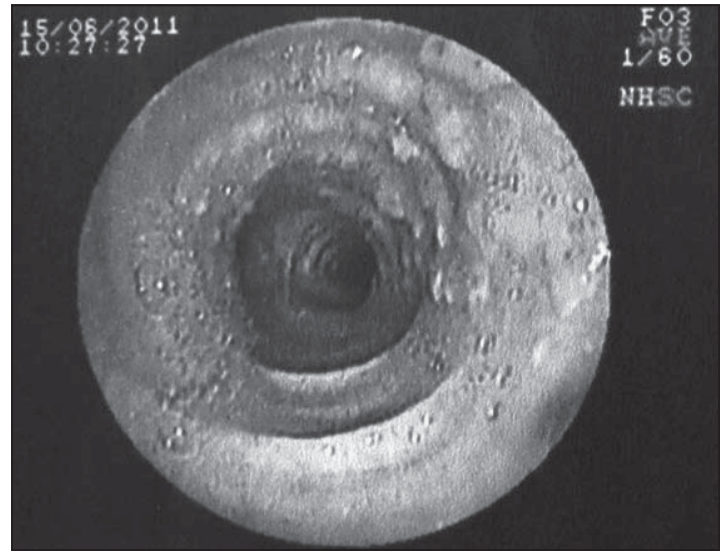

Figura 7. Videobroncoscopia: “Jardín de rocas” o “adoquinado” en paredes anterior y lateral de la tráquea.

genética ${ }^{5}$; se asocia frecuentemente a inflamación crónica infecciosa (Mycobacterium avium intracellulare, Kebsiella ozena) o química de las vías aéreas superiores e inferiores (exposición a sílice, reflujo gastroesofágico) $)^{6,7}$ aunque no se puede aseverar si la asociación es causa o consecuencia; con respecto a esto último, se ha reportado una alteración severa del clearance mucociliar en pacientes portadores de TOCP, la cual influye negativamente en la efectividad de los mecanismos de defensa inespecíficos del árbol traqueobronquial, hecho que los hace susceptibles a infecciones respiratorias repetidas ${ }^{8}$. Nuestra primera paciente sufría de infecciones respiratorias reiteradas, siendo portadora de una rinitis atrófica con ocena y reflujo gastroesofágico.

Se le relaciona también con alteraciones metabólicas (amiloidosis), procesos degenerativos o metaplasia y posterior transformación en cartílago y osificación del tejido elástico. Se ha comunicado asociación con linfoma de Hodgkin y no Hodgkin y cáncer epidermoide del pulmón; cáncer de tiroides y de mama, pero no hemos encontrado reportes de mesotelioma coincidiendo con el hallazgo de TOCP como en nuestro segundo caso.

Existe evidencia histológica que apoya la acción sinérgica de la proteína morfogenética de hueso 2 (BMP-2) con el factor transformador de crecimiento Beta1 (TGFß1) en la formación de los nódulos óseos y/o cartilaginosos, característicos de la entidad en la submucosa traqueal. La BMP-2 es un miembro de la superfamilia de genes que expresan el TGF- $\beta 1$ que induce la neoformación de hueso y cartílago traqueal. En material de autopsia de pacientes portadores de TOCP se encontró inmunorreactividad positiva para BMP-2 en las células mesenquimales y en 
los condroblastos que rodeaban los nódulos de la submucosa traqueal y para TGF- $\beta 1$ en los condrocitos y osteocitos de dichos nódulos ${ }^{9}$.

La enfermedad afecta a ambos sexos, habiendo una ligera predominancia en varones, con una relación 1:3 mujer/hombre. La mayoría de los casos descritos se diagnostican alrededor de los 50 años pero se ha encontrado también en niños de hasta 6 años de edad ${ }^{10,11}$. Es probable que las alteraciones anatómicas comiencen a edades más tempranas, siendo el cuadro asintomático y por lo tanto subdiagnosticado durante muchos años. No existe una sintomatología específica pero al momento del diagnóstico, cerca del 88\% de los pacientes presentan tos crónica que no cede a la terapia habitual, disnea de esfuerzos medianos que puede no ser progresiva y que se relaciona con infecciones repetidas de las vías aérea, pero que no cede al tratamiento; hemoptisis en general de pequeña magnitud y estridor, cuando se produce deformidad importante u obstrucción severa de la tráquea por los nódulos que protruyen hacia el lumen. Frecuentemente se le confunde y trata como asma refractaria ${ }^{12-14}$. La función respiratoria, cuando se compromete, revela un patrón restrictivo en la espirometría o una alteración obstructiva fija de vía aérea central en la curva flujo/volumen ${ }^{15,16}$ como encontramos en el primer caso. La evolución en el tiempo es benigna, pero en ocasiones progresa hasta causar falla respiratoria severa ${ }^{17}$.

La imagenología y la endoscopía son diagnósticas. La radiografía simple de tórax muestra alteraciones inespecíficas, pero la tomografía computada puede mostrar imágenes nodulares calcificadas que protruyen desde la submucosa traqueal o bronquial hacia el lumen, siempre, respetando la pared membranosa o un aplastamiento lateral de las paredes traqueales conformando una imagen en hoja de sable ${ }^{18}$. Útil es la broncoscopía virtual a partir de imágenes de tomografía computada, cuyos hallazgos se corresponden bien con la broncoscopía tradicional, sin ser invasiva ${ }^{19}$.

Endoscópicamente hay múltiples nódulos submucosos de aspecto blanquecino habitualmente localizados en el tercio inferior de la tráquea y en bronquios principales, menos frecuente en la región subglótica y en bronquios lobares, comprometiendo exclusivamente las paredes laterales y anterior, hallazgo importante que sirve para diferenciar el cuadro de otras patologías como amiloidosis, metástasis endobronquiales o infecciones que no respetan la pared posterior. Los nódulos descritos en ocasiones coalescen llegando a formar cúmulos que se describen como "jardín de rocas”. Dichas lesiones pueden obstruir gran parte del lumen de la vía aérea comprometida haciendo difícil el paso hacia distal del broncoscopio. Al tacto con la pinza son duras y por lo mismo es difícil obtener material adecuado de tejido para examen histológico ${ }^{20}$. En el primer caso se observó compromiso desde el tercio medio de la tráquea hasta ramos subsegmentarios, lo que constituye una excepción y lo hace diferente a los casos comunicados en la literatura a que tuvimos acceso. En esta paciente, un primer examen endoscópico realizado 19 años antes mostraba compromiso avanzado, pero sólo hasta bronquios lobares, de modo que es probable que en el tiempo las lesiones se extiendan lentamente en sentido distal hasta comprometer el resto de la vía aérea de conducción. En la literatura internacional hay pocos casos con un seguimiento tan largo, de modo que es nuestra impresión que este caso ilustra bien la evolución natural de la enfermedad.

Histológicamente hay proliferación de cartílago con depósitos de calcio en la submucosa y formación de tejido óseo. La mucosa que cubre las lesiones no muestra alteraciones y su epitelio es normal, tal como se observa en la Figura 5-A de nuestro primer caso. Ocasionalmente, puede haber fragmentos de tejido adiposo con focos de médula ósea con hematopoyesis activa ${ }^{21,22}$. En la Figura 5-B se observa tejido osteocartilaginoso en transición hacia la maduración, con actividad osteoblástica y espacios medulares.

En el diagnóstico diferencial se debe incluir a la amiloidosis, sarcoidosis, lesiones secuelares de tuberculosis calcificada, papilomatosis traqueobronquial y tumores de la vía aérea. La visión endoscópica es fundamental ya que es una característica exclusiva de la TOCP el respeto de la pared membranosa de tráquea y bronquios hecho que no sucede en ninguno de los cuadros mencionados ${ }^{22}$.

El manejo es conservador pues pocas veces al momento del diagnóstico las lesiones son localizadas. Se ha intentado resecciones de segmentos más comprometidos y terapia endoscópica con láser y crioterapia, con resultados variables ${ }^{21}$. Hay comunicaciones del uso de radioterapia externa en un número limitado de casos. Dado que la alteración anatómica produce deformidades rígidas, es difícil la dilatación e instalación de stent traqueobronquiales. El tratamiento médico es pobre y se limita a la mantención de la humedad de la vía aérea y al pronto tratamiento de las infecciones. No hay respuesta al tratamiento broncodilatador ni antiinflamatorio. Diferentes técnicas quirúrgicas se han empleado, entre ellas, la traqueoplastía con resección oblicua del segmento traqueal comprometido, que permite aumentar el diámetro traqueal sin lesión isquémica secundaria 
al procedimiento ${ }^{23}$. Hasta el momento, la técnica quirúrgica que mejores resultados ha obtenido es la traqueoplastía lineal, con incisión anterior de la tráquea hasta la carina incluyendo o no los bronquios fuente, con la posterior instalación intraluminal por tiempo prolongado de una prótesis recta o en Y. La incisión longitudinal de la pared anterior de la tráquea, permite la apertura de las paredes laterales al recogerse la porción membranosa, la mantención del lumen con la prótesis intraluminal permite que al cicatrizar la incisión, se mantenga un lumen más amplio lo que elimina en parte la sintomatología cuando ésta es severa $^{18,24}$. No conociéndose la etiopatogenia de la enfermedad, no se puede plantear una terapia racional que se dirija a eliminar factores causales, o a bloquear o estimular determinados mediadores que impidan la progresión de las lesiones del árbol traqueobronquial. Es muy probable que con el aumento en las expectativas de vida de la población, los casos diagnosticados aumenten en número y se haga imprescindible encontrar algún tipo de terapia efectiva.

\section{Conclusiones}

Las dos pacientes presentadas ilustran bien las características clínicas, imagenológicas, broncoscópicas e histológicas de la TOCP, junto a las alteraciones funcionales en una de ellas y su curso lentamente progresivo que, indefectiblemente llevará en algún momento a la aparición de síntomas respiratorios que no remiten con el tratamiento por lo que, la presencia de ellos, especialmente tos y/o hemoptisis sin una explicación clara, obliga a descartarla y a buscar las condiciones asociadas a la misma.

\section{Referencias bibliográficas}

1.- MOLLOY A R, MCMAHON J N. Rapid progression of tracheal stenosis associated with tracheopathia osteochondroplastica. Intensive Care Med 1988; 15: 60-2.

2.- THOMAS D, STONELL C, HASAN K. Tracheobronchopathia osteoplastica: incidental finding at tracheal intubation. Br J Anaesth 2001; 87: 515-7.

3.- DÍAZ J C, RUIZ M. Traqueobroncopatía osteocondroplástica como causa de vía aérea difícil. Rev Chil Med Intensiva 2008; 23: 263-4.

4.- HUSSAIN K, GILBERT S. Tracheopathia osteochondroplastica. Clin Med Res 2003; 1: 239-42.

5.- PRAKASH U B, MCCULLOUGH A E, EDELL E S, NIENHUIS D M. Tracheopathia osteoplastica: familial occurrence. Mayo Clin Proc 1989; 64:1091-6.
6.- BAUGNEE P E, DELAUNOIS L S. Mycobacterium avium-intracellulare associated with tracheobronchopathia osteochondroplastica. Eur Respir J 1995; 8: 1802.

7.- MAGRO P, GARAND G, CATTIER B, RENJARD L, MARQUETTE C H, DIOT P. Association of tracheobronchopathia osteochondroplastica and ozène. Rev Mal Respir 2007; 24: 883-7.

8.- CHEN A Y, DONOVAN D T. Impaired ciliary clearance from tracheopathia osteoplastica of the upper respiratory tract. Otolaryngol Head Neck Surg 1997; 117: S102-4.

9.- TAJIMA K, YAMAKAWA M, KATAGIRI T, SASAKI H. Immunohistochemical detection of bone morphogenetic protein-2 and transforming growth factor beta-1 in tracheopathia osteochondroplastica. Virchows Arch 1997; 431: 359-63.

10.- SIMSEK P O, OZCELIK U, DEMIRKAZIK F, UNAL O F, ORHAN D, ASLAN A T, et al. Tracheobronchopathia osteochondroplastica in a 9-year-old girl. Pediatr Pulmonol 2006; 41: 95-7.

11.- MARTIN C J. Tracheobronchopathia osteochondroplastica. Arch Otolaryngol 1974; 100: 290-3.

12.- DECALMER S, WOODCOCK A, GREAVES M, HOWE M, SMITH J. Airway abnormalities at flexible bronchoscopy in patients with chronic cough. Eur Respir J 2007; 30: 1138-42.

13.- HAYES D Jr. Tracheopathia osteoplastica misdiagnosed as asthma. J Asthma 2007; 44: 253-5.

14.- WILLMS H, WIECHMANN V, SACK U, GILLISSEN A. Tracheobronchopathia osteochondroplastica: A rare cause of chronic cough with haemoptysis. Cough 2008; 30: 4: 4.

15.- LUNDGREN R, STJERNBERG N L. Tracheobronchopatia osteochondroplastica. A clinical, bronchoscopy and spirometric study. Chest 1981; 80: 706-9.

16.- TUKIAINEN H, TORKKO M, TERHO E O. Lung function in patients with tracheobronchopathia osteochondroplastica. Eur Respir J 1988; 1: 632-5.

17.- MBOTI FB, NINANE V, LARSIMONT D, LEURQUIN M, LEMORT M, CHASSAING C, et al. Acute respiratory failure from tracheopathia osteoplastica. Acta Chir Belg 2005; 105: 104-5.

18.- GRILLO H C, WRIGHT C D. Airway obstruction owing to tracheopathia osteoplastica: treatment by linear tracheoplasty. Ann Thorac Surg 2005; 79: 1676-81.

19.- HUANG C C, KUO C C. Chronic cough: tracheobronchopathia osteochondroplastica. CMAJ 2010; 14; 182 : E859.

20.- YOKOYAMA T, FUJIYOSHI Y, NINOMIYA H, TASHIRO K, KAJIKI A, TAKAMOTO M, et al. Tracheobronchopathia osteoplastica with "rock garden" findings on bronchoscopy. Nihon Kyobu Shikkan Gakkai Zasshi 1997; 35: 477-80.

21.- JABBARDARJANI H R, RADPEY B, KHARABIAN S, MASJEDI M R. Tracheobronchopathia osteochon- 
droplastica: presentation of ten cases and review of the literature. Lung 2008; 186: 293-7.

22.- CHRONEOU N, ZIAS A V, GONZÁLEZ J F, BEAMIS

JR. Introduction. Tracheobronchopathia osteochondroplastica. An underrecognized entity? Monaldi Arch Chest Dis 2008; 69: 65-9.

23.- KUTLU C A, YEGINSU A, OZALP T, BARAN R.
Modified slide tracheoplasty for the management of tracheobronchopathia osteochondroplastica. Eur J Cardiothorac Surg 2002; 21: 140-2.

24.- CELEDON C, DE GRAZIA J. Traqueobroncopatía osteocondroplástica: reporte de un caso y revisión de la literatura. Rev. Otorrinolaringol. Cir Cabeza Cuello 2010; 70: 147-52.

Correspondencia a:

Dr. Patricio Rioseco S.

Médico Broncopulmonar

Hospital Las Higueras

Servicio de Medicina

Alto Horno \# 777 - Concepción.

E-mail: parioseco@gmail.com

Rev Chil Enf Respir 2012; 28: 143-149 